\title{
LIMITS ON STRINGY SIGNALS AT THE LHC
}

\author{
DIETER LÜST \\ Max-Planck-Institut für Physik, \\ Werner-Heisenberg-Institut, 80805 München, Germany \\ Arnold Sommerfeld Center for Theoretical Physics Ludwig-Maximilians-Universität München, \\ 80333 München, Germany \\ TOMASZ R. TAYLOR \\ Department of Physics, \\ Northeastern University, Boston, MA 02115, USA
}

\begin{abstract}
We explain what features of string theory can be tested at the LHC. We review the present bounds on the string mass and on extra gauge bosons as well as the prospects for the upcoming experiments.

Keywords: LHC, low mass strings, extra dimensions
\end{abstract}

\section{Introduction}

After the discovery of the Higgs-like particle with mass around $125 \mathrm{GeV}$ by the ATLAS and CMS collaborations at CERN, still many convincing reasons exist to believe that the resolution of the hierarchy problem lies in new physics around the $\mathrm{TeV}$ mass scale. In fact, there are at least two, not necessarily mutually exclusive scenarios, offered as solutions of the hierarchy problem:

- Low energy supersymmetry at around $1 \mathrm{TeV}$.

- Large extra dimensions and a low scale for quantum gravity at around $1 \mathrm{TeV} 112$.

Here we discuss some rather generic features about string and brane compactifications, which are relevant for the search of new physics at the LHC and which might provide concrete and fundamental realizations of the general scenarios mentioned above. In fact, the relevance of strings for the experiments at the LHC is closely linked to the question what is the typical scale of string theory, called the string mass $M$. A priori, considering D-brane compactifications with open strings on branes, the string scale is a free parameter, not being determined by the Standard Model gauge interactions. Hence in this class of models $M$ can be in principle everywhere between the weak scale and the Planck scale. Therefore we divide the discussion into two cases, namely into low and high string scale compactifications. 


\subsection{Low string scale compactifications}

The most spectacular and most direct LHC signatures from strings will emerge in case $M$ is very low, namely at the order of $\mathrm{TeV}$. Then the gravitational and gauge interactions are unified at around $1 \mathrm{TeV}$, and the observed weakness of gravity at lower energies is due to the existence of large extra dimensions, where the volume $V$ of the internal space in units of $M$ must be of the order $V M^{6}=\mathcal{O}\left(10^{32}\right)$. This scenario can be nicely realized by D-brane compactifications (for a review see e.g. [3]). Here gravitons, i.e. closed strings may scatter into the extra space and by this the gravitational coupling constant is decreased to its observed value. On the other hand, the particles of the Standard Model correspond to the zero modes of open strings that are confined to lower dimensional D-branes, which typically fill part of the extra-dimensions and also intersect among each other in the higher dimensional space. A typical brane configuration $\frac{4}{4}$ is shown in Fig.1 ${ }^{5}$ Gauge bosons emerge

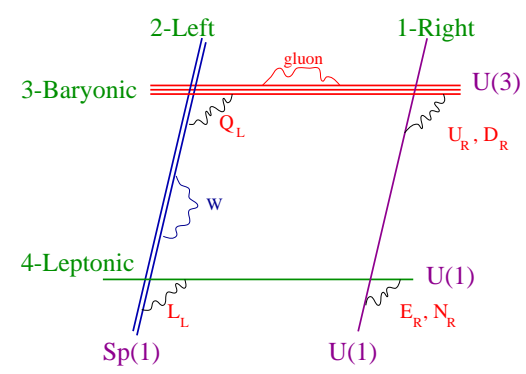

Fig. 1. A typical configuration of intersecting branes, in this case corresponding to the so-called $\mathrm{SM}^{++}$model with the gauge group $S U(3) \times S U(2) \times U(1)_{Y} \times U(1)^{2}$.

from open strings ending on a the same stack of overlapping branes, with $\mathrm{U}(1)$ gauge bosons ending on the same brane, while non-abelian gauge bosons like gluons stretch between distinct branes in a single stack. Quarks and leptons emerge from strings stretching between intersecting stacks of branes. Moreover a whole tower of massive open string excitations will open up at around $1 \mathrm{TeV}$, where the new particles follow the well known Regge "trajectories" of vibrating strings,

$$
M_{n}^{2}=n M^{2}, \quad j=j_{0}+\alpha^{\prime} M_{n}^{2},
$$

with spin $j$ particles populating lines with the universal slope $\alpha^{\prime}$ determined by the fundamental string mass scale $M^{2}=\alpha^{\prime-1}$, and "intercepts" $j_{0}$. At the $n$-th level, the spins of excited gluons range from 0 to $n+1$ and of excited quarks from $1 / 2$ to $n+1 / 2$. The standard model spectrum is replicated at each level $n$ by higher spin particles.

Let us list what kind of string signatures from a low string scale and from large extra dimensions in brane models can be possibly expected at the LHC:

(i) The discovery of string Regge excitations with masses of order $M$. As we will 
explain in the following, the production cross sections and the decay rates of the heavy string excitations can be computed in a model independent way for a large class of D-brane models, such that universal predictions for the discovery of these particles can be made. In fact, the production of string Regge excitations will lead to new contributions to standard model scattering processes, like QCD jets or scattering of quarks into leptons or gauge bosons, which can be measurable at LHC in case the string scale is low $6 / 7 / 8|9| 10|11| 12$.

(ii) The discovery of new exotic particles around $M$. Another very interesting signal for new stringy physics at the LHC is the production of heavy neutral $Z^{\prime}$ gauge bosons (see e.g. [13, 14, 15, 16]). These particles are quite generic in any string compactification, and they receive their mass via a Green-Schwarz mixing with axionic scalar fields. E.g. in a four stack D6-brane model with gauge group $S U(3) \times$ $S U(2) \times U(1)_{Y} \times U(1)^{2}$, see Fig.1, at least one extra $U(1)$ gauge bosons will get a mass of order $g^{\prime} M<M$ by the Green-Schwarz effect.

(iii) The discovery of (non-perturbative) quantum gravity effects in the form of mini black holes. One of the most exciting possibilities for the LHC is the discovery of small higher-dimensional black holes that can be formed when two sufficiently energetic particles collide $17|18| 19 \mid 20$. This means that effects of higher dimensional quantum gravity can get strong if the string scale is low around the $\mathrm{TeV}$ scale, and if the volume of the extra dimensions is large. The geometrical cross section for the production of mini black holes is of the order

$$
\sigma(E) \sim \frac{1}{M_{b . h .}^{2}}\left(\frac{E}{M_{b . h .}}\right)^{\alpha},
$$

where $M_{b . h .}$. is the black hole mass, i.e. the effective scale of quantum gravity, and $\alpha \leq 1$ for higher dimensional black holes. Since the production of mini black holes is basically a non-perturbative effect, the black hole mass is suppressed by the string coupling constant compared to the string scale:

$$
M_{b . h .} \sim \frac{M}{g_{\text {string }}} .
$$

Therefore, for weak string coupling, the threshold for black hole production is almost inevitably higher than the threshold for the production of string resonances.

(iv) The discovery of Kaluza-Klein (KK) excitations. In superstring theory with D-branes there are two classes of Kaluza-Klein states: bulk excitations of closed string states - like KK graviton etc. - and the excitations associated to the extra dimensions of D-branes extending into the bulk space. The masses of bulk states are determined by the properties of the six-dimensional Calabi-Yau manifold. The KK excitations of D-brane states are charged under the respective gauge group and their masses depend on how D-branes wrap into closed surfaces (cycles) inside Calabi-Yau manifold. Experimental signatures of KK states are common to all extra- 
dimensional extensions of the standard model and will be discussed elsewhere in this volume.

\subsection{High string scale compactifications}

Now let us turn to string compactifications with the string scale much above the $\mathrm{TeV}$ region. In fact, there are no compelling reasons why the string mass scale should be much lower than the Planck mass. In the large volume compactifications of Refs. 21, 22, 23] it was shown that that one can stabilize moduli in such a way that the string scale $M$ is at intermediate energies of about $10^{11-12} \mathrm{GeV}$. Then the internal $\mathrm{CY}$ volume $V$ is of order $V M^{6}=\mathcal{O}\left(10^{16}\right)$. The motivation for this scenario is to obtain a low supersymmetry breaking scale around $1 \mathrm{TeV}$, since one derives the following relation for the gravitino mass:

$$
m_{3 / 2} \sim \frac{M^{2}}{M_{\text {Planck }}}
$$

Hence the experimental indications for this kind of scenario at the LHC would be given by usual signatures for low energy supersymmetry, whose details largely depend on further assumptions of the concrete compactification scheme.

Finally the string scale can be also around the Planck scale or also a bit lower around the GUT scale of about $10^{16} \mathrm{GeV}$, a choice, which is motivated by the unification of the gauge coupling constants of the Standard Model. However Dbrane models with such a high string scale can nevertheless lead to some rather model independent new physics predictions around the TeV scale. Specifically, as discussed in [5], in generic 4-stack D-brane models one typically arrives at a simple extension of the Standard Model, called $\mathrm{SM}^{++}$(see Fig.1) obtained by adding to the scalar sector a complex $S U(2)$ singlet that has the quantum numbers of the right-handed neutrino, $H^{\prime \prime}$, and to the gauge sector an $U(1)$ that is broken by the vacuum expectation value of $H^{\prime \prime}$ (see also [24]). The associated $Z^{\prime \prime}$-gauge boson can be relatively light.

\section{Low string scale: string resonances in dijets}

The lightest string resonances are excited quarks and gluons which should be copiously produced at the LHC energies just above the string threshold. They are produced by gluon-gluon or quark-gluon fusion, at the rates typical for QCD processes. These resonances decay into gluon pairs or quarks and gluons, giving rise to spectacular resonance bumps in dijet cross sections. As an example, consider the lightest quark resonances $q^{*}$, which appear in two separate spin $j=1 / 2$ and $j=3 / 2$ eigenstates. They give rise to a peak in the squared modulus of the quark- 
gluon scattering amplitude, at the center of mass energies close to their mass $M$ :

$$
\begin{aligned}
|\mathcal{M}(q g \rightarrow q g)|^{2}=-\frac{4}{9} \frac{g^{4}}{M^{2}} & {\left[\frac{M^{4} \hat{u}}{\left(\hat{s}-M^{2}\right)^{2}+\left(\Gamma_{q^{*}}^{j=1 / 2} M\right)^{2}}\right.} \\
& \left.+\frac{\hat{u}^{3}}{\left(\hat{s}-M^{2}\right)^{2}+\left(\Gamma_{q^{*}}^{j=3 / 2} M\right)^{2}}\right],
\end{aligned}
$$

where $\hat{s}$ and $\hat{u}$ are the Mandelstam invariants of this particular subprocess, and $g$ is the QCD coupling constant $\left(\alpha_{\mathrm{QCD}}=\frac{g^{2}}{4 \pi} \approx 0.1\right)$. Here, the resonance poles have been softened to a Breit-Wigner form with the partial widths $\Gamma_{q^{*}}^{j=1 / 2}=\Gamma_{q^{*}}^{j=3 / 2}=$ $37(M / \mathrm{TeV}) \mathrm{GeV}$, as calculated in Ref. 25. The squared amplitudes for all $2 \rightarrow 2$ and $2 \rightarrow 3$ parton subprocesses are collected in Refs. 811] while the decay rates can be found in Ref. 25]. It is remarkable that the amplitude (5), as well as the amplitude describing $g g \rightarrow g g$ scattering, do not contain any reference to extra dimensions. Recall that one of the most common criticisms of string theory is that it does not predict the shape or size of extra dimensions responsible for the properties of low energy effective theory - usually called the landscape problem - but here, this problem has been circumvented by a complete universality of the amplitudes. As long as quark and gluons appear in the low energy spectrum, string resonances contribute in a universal way, common to all compactifications. There are conceivably no other extensions of the standard model that would produce such unambiguous, spectacular signatures of strongly interacting, higher spin particles. In reality, string resonances are very easy to discover.

The amplitudes involving four and more fermions, like $q \bar{q} \rightarrow q \bar{q}$, do depend on the details of compactification, however they do not significantly contribute to dijet cross sections for two reasons: 1) color triplets yield smaller $S U(3)$ QCD factors than gluon octets 2) at high energies (large Feynman's $x$ ), antiquark lumnosities inside protons are lower than quark and gluon luminosities. Actual dijet mass distributions are obtained by convoluting parton cross sections with the proton structure functions. As seen in Fig.2 $\frac{9}{9} q g \rightarrow q g$ is the most favorable channel for discovering string resonances, with an optimal balance of luminosity and color factors.

No significant bumps in dijet mass distributions have been observed yet at the LHC in the initial runs at $\sqrt{s}=7 \mathrm{TeV}$ and $\sqrt{s}=8 \mathrm{TeV}$. The results of CMS searches for string resonances, based on the integrated luminosity of $4 \mathrm{fb}^{-1}$ at $\sqrt{s}=$ $8 \mathrm{TeV}$, are shown on Fig.3.26 The data are presented as the observed upper limits (at $95 \%$ confidence level) on $\sigma \times \mathcal{B} \times A$ where $\mathcal{B} \approx 1$ is the branching fraction of $q^{*}$ into $q g$ and $A \approx 0.6$ is the acceptance for the respective kinematic requirements. The cross section $\sigma$ is integrated over the mass bins determined by the uncertainty in jet energy resolution 3 Based on these data, CMS quote the lower bound, $M>4.78$

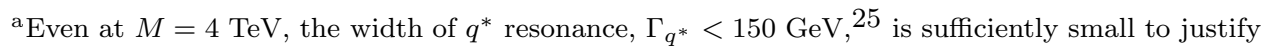
the narrow resonance approximation applied to CMS data. 


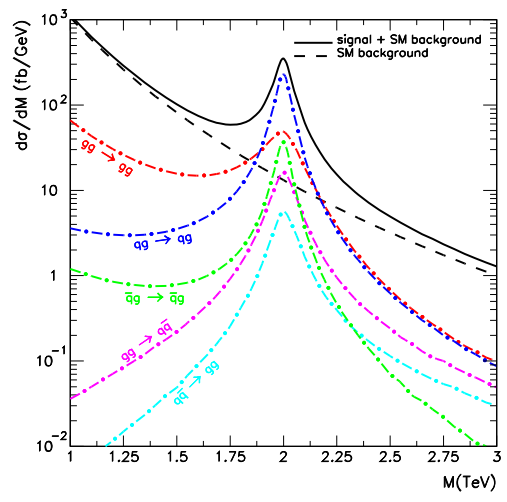

Fig. 2. A peak in the dijet cross section $d \sigma / d M$ (units of $\mathrm{fb} / \mathrm{GeV}$ ) vs. $\mathrm{M}(\mathrm{TeV}$ ) is plotted for the case of SM QCD background (dashed line) and (first resonance) string signal + background (solid line), for the hypothetical (already excluded, see below) string mass value of $2 \mathrm{TeV}$ and LHC $\sqrt{s}=14 \mathrm{TeV}$. The dot-dashed lines indicate contributions of individual channels, illustrating the dominance of $q g \rightarrow q g$.

$\mathrm{TeV}$, on the string mass

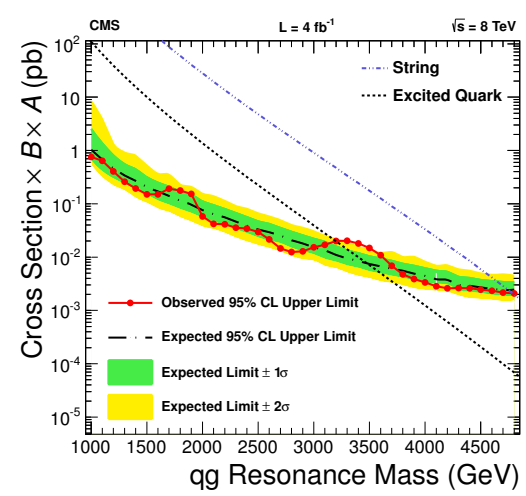

Fig. 3. Observed upper limits on $\sigma \times \mathcal{B} \times A$ for resonances decaying into $q g$ final state (points and solid lines). The predicted cross section, marked as "String", is based on the assumption of $q^{*} j=1 / 2$ and $j=3 / 2$ resonances.

Low mass string theory will get another chance in the next run. As seen in Fig.4,$\frac{9}{9}$ string mass scales as high as 7 or even $8 \mathrm{TeV}$ can be reached by LHC operating at its design center of mass energy $\sqrt{s}=14 \mathrm{TeV}$.

${ }^{\mathrm{b}} \mathrm{CMS}$ has also ruled out excited top quarks with $M_{t^{*}}<790 \mathrm{GeV}$ by searching in the top quark plus gluon decay channels 27 This provides an additional bound on a class of "warped" string compactifications 28 


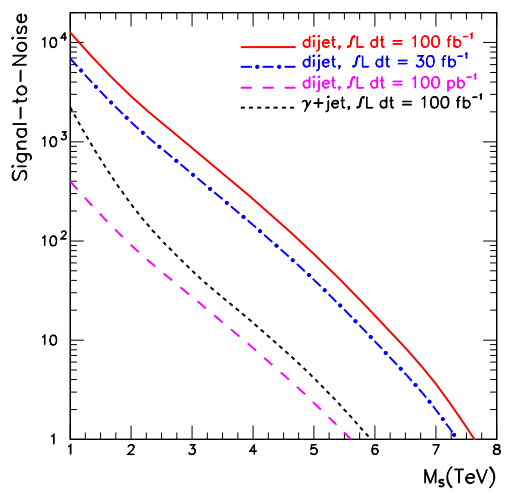

Fig. 4. Dijet signal-to-noise ratio for string resonances at the LHC with the design $\sqrt{s}=14 \mathrm{TeV}$, for three integrated luminosities.

\section{High (and low) string scale: extra $U(1)$ gauge bosons}

No matter what the string scale is, all D-brane models predict the existence of more neutral gauge bosons than the standard model. The reason is that gauge groups are associated to stacks of D-branes and in this framework, the color $S U(3)$ group emerges from a stack of at least three branes which, in the minimal case, generate $U(3)=S U(3) \times U(1)$, with the extra $U(1)$ coupled to the baryon number $B$. Since $B$ is an anomalous symmetry, the corresponding $Z^{\prime}$ gauge boson receives a mass via Green-Schwarz mechanism, of order $g^{\prime} M<M$. In general, there are more than one extra gauge bosons. They fall into two classes: anomalous, massive $Z^{\prime}$ or non-anomalous $Z^{\prime \prime}$ with the mass generated by vacuum expectation values of an extended Higgs sector. In the latter case, there is no direct relation between the mass and the string scale $M$.

There are many other extensions of the standard model involving extra gauge bosons, so such a particle would constitute at most a "harbinger" of string theory. It is natural to expect that $Z^{\prime}$ and/or $Z^{\prime \prime}$ couple to quarks: in this case a bump would be observed in the dijet invariant mass spectrum. Unfortunately, no extra gauge bosons have been found yet at the LHC, see Fig.5.26 In this case, CMS collaboration quotes $M_{Z^{\prime}}>1.62 \mathrm{TeV}$ assuming that $Z^{\prime}$ couples with the same strength as $Z$.

If the string mass is too high for the string resonances or $Z^{\prime}$ to be discovered at the LHC, $Z^{\prime \prime}$ gauge bosons, with masses determined by an extended Higgs sector, still remain as a possibility. In a minimal model of that type, called $\mathrm{SM}^{++}, 5$ one additional D-brane intersects the $U(3)$ stack. The non-anomalous $Z^{\prime \prime}$ couples to the combination of $B-L$ ( $L$ is the lepton number) and the right-handed isospin $I_{R}$. The strength of its $q \bar{q}$ coupling depends on the combination, and the existing dijet data can be interpreted in this context to establish more specific bounds on $Z^{\prime \prime}$ mass, as seen in Fig.6. $\frac{5}{5}$ The LHC, operating at its design center of mass energy, will explore 


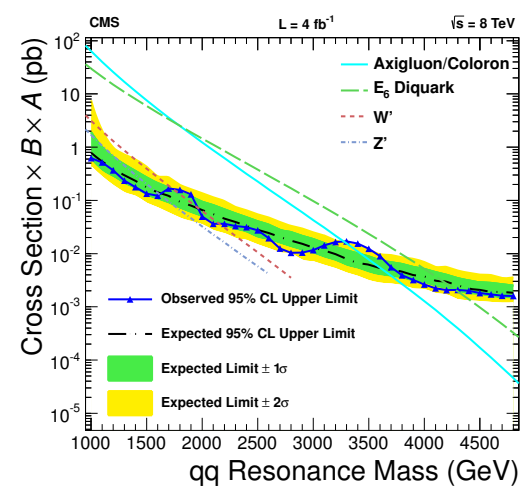

Fig. 5. Limits on various resonances, including $Z^{\prime}$, decaying into $q \bar{q}$ final state.
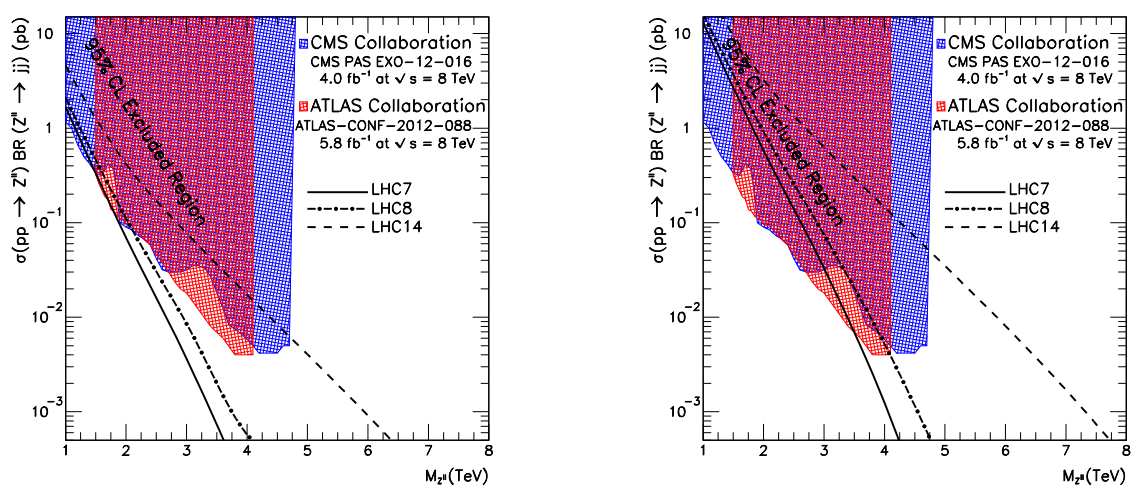

Fig. 6. Comparison of the total cross section for the production of $p p \rightarrow Z^{\prime \prime} \rightarrow j j$ with the $95 \%$ CL upper limits on the production of a gauge boson decaying into two jets as reported by the CMS and ATLAS collaborations (corrected by acceptance). The case in which $Z^{\prime \prime}$ is mostly diagonal in $I_{R}$ is shown in the left panel and the case in which it is mostly $B-L$ in the right panel.

a wider range of $Z^{\prime \prime}$ masses.

\section{Conclusions}

No low mass string or the associated extra gauge bosons have been observed in the LHC runs at $\sqrt{s}=7 \mathrm{TeV}$ and $\sqrt{s}=8 \mathrm{TeV}$. Unlike in many other beyond the standard model extensions, due to spectacular strength of dijet signals, low mass strings will be either discovered at the early stage of the next run or more stringent bounds will be established very soon. With the present bound $M>4.78 \mathrm{TeV}$, the best we can hope for is the discovery of the lowest, $n=1$ string excitations of quarks and gluons because higher levels and the accompanying mini black holes will be most likely beyond the reach of LHC. Nevertheless, with such large cross sections, it should be possible to identify higher spin string excitations of gauge 
bosons, quarks and leptons.

\section{Acknowledgments}

This work was partially supported by the ERC Advanced Grant "Strings and Gravity" (Grant.No. 32004) and by the DFG cluster of excellence "Origin and Structure of the Universe". In addition, this material is based in part upon work supported by the National Science Foundation under Grant No. PHY-0757959. Any opinions, findings, and conclusions or recommendations expressed in this material are those of the authors and do not necessarily reflect the views of the National Science Foundation.

\section{References}

1. N. Arkani-Hamed, S. Dimopoulos and G. R. Dvali, "The Hierarchy problem and new dimensions at a millimeter," Phys. Lett. B 429, 263 (1998) hep-ph/9803315.

2. I. Antoniadis, N. Arkani-Hamed, S. Dimopoulos and G. R. Dvali, "New dimensions at a millimeter to a Fermi and superstrings at a TeV," Phys. Lett. B 436, 257 (1998) hep-ph/9804398.

3. R. Blumenhagen, B. Körs, D. Lüst, S. Stieberger, "Four-dimensional String Compactifications with D-Branes, Orientifolds Phys. Rept. 445, 1 (2007) hep-th/0610327.

4. D. Cremades, L. E. Ibanez and F. Marchesano, "Yukawa couplings in intersecting D-brane models," JHEP 0307, 038 (2003) hep-th/0302105.

5. L. A. Anchordoqui, I. Antoniadis, H. Goldberg, X. Huang, D. Lüst, T. R. Taylor and B. Vlcek, "LHC Phenomenology and Cosmology of String-Inspired Intersecting D-Brane Models," Phys. Rev. D 86, 066004 (2012) arXiv:1206.2537 [hep-ph]].

6. S. Cullen, M. Perelstein and M. E. Peskin, "TeV strings and collider probes of large extra dimensions," Phys. Rev. D 62 (2000) 055012 hep-ph/0001166.

7. D. Chialva, R. Iengo and J. G. Russo, "Cross sections for production of closed superstrings at high energy colliders in brane world models," Phys. Rev. D 71 (2005) 106009 hep-ph/0503125.

8. D. Lüst, S. Stieberger and T. R. Taylor, "The LHC String Hunter's Companion," Nucl. Phys. B 808, 1 (2009) arXiv:0807.3333 [hep-th]].

9. L. A. Anchordoqui, H. Goldberg, D. Lüst, S. Nawata, S. Stieberger and T. R. Taylor, "Dijet signals for low mass strings at the LHC," Phys. Rev. Lett. 101, 241803 (2008) arXiv:0808.0497 [hep-ph]].

10. L. A. Anchordoqui, H. Goldberg, D. Lüst, S. Nawata, S. Stieberger and T. R. Taylor, "LHC Phenomenology for String Hunters," Nucl. Phys. B 821 (2009) 181 arXiv:0904.3547 [hep-ph]].

11. D. Lüst, O. Schlotterer, S. Stieberger and T. R. Taylor, "The LHC String Hunter's Companion (II): Five-Particle Amplitudes and Universal Properties," Nucl. Phys. B 828, 139 (2010) arXiv:0908.0409 [hep-th]].

12. Z. Dong, T. Han, M. -x. Huang and G. Shiu, "Top Quarks as a Window to String Resonances," JHEP 1009, 048 (2010) arXiv:1004.5441 [hep-ph]].

13. E. Kiritsis and P. Anastasopoulos, "The Anomalous magnetic moment of the muon in the D-brane realization of the standard model," JHEP 0205 (2002) 054 hep-ph/0201295.

14. D. M. Ghilencea, L. E. Ibanez, N. Irges and F. Quevedo, "TeV scale Z-prime bosons from D-branes," JHEP 0208 (2002) 016 hep-ph/0205083. 
15. L. A. Anchordoqui, H. Goldberg, X. Huang, D. Lüst and T. R. Taylor, "Stringy origin of Tevatron Wjj anomaly," Phys. Lett. B 701, 224 (2011) arXiv:1104.2302 [hep-ph]].

16. L. A. Anchordoqui, I. Antoniadis, H. Goldberg, X. Huang, D. Lüst and T. R. Taylor, "Z'-gauge Bosons as Harbingers of Low Mass Strings," Phys. Rev. D 85, 086003 (2012) arXiv:1107.4309 [hep-ph]].

17. T. Banks and W. Fischler, "A Model for high-energy scattering in quantum gravity," hep-th/9906038.

18. S. B. Giddings and S. D. Thomas, "High-energy colliders as black hole factories: The End of short distance physics," Phys. Rev. D 65 (2002) 056010 hep-ph/0106219.

19. S. Dimopoulos and G. L. Landsberg, "Black holes at the LHC," Phys. Rev. Lett. 87 (2001) 161602 hep-ph/0106295.

20. P. Meade and L. Randall, "Black Holes and Quantum Gravity at the LHC," JHEP 0805 (2008) 003 arXiv:0708.3017 [hep-ph]].

21. V. Balasubramanian, P. Berglund, J. P. Conlon and F. Quevedo, "Systematics of moduli stabilisation in Calabi-Yau flux compactifications," JHEP 0503 (2005) 007 hep-th/0502058.

22. J. P. Conlon, F. Quevedo and K. Suruliz, "Large-volume flux compactifications: Moduli spectrum and D3/D7 soft supersymmetry breaking," JHEP 0508 (2005) 007 hep-th/0505076.

23. J. P. Conlon, C. H. Kom, K. Suruliz, B. C. Allanach and F. Quevedo, "Sparticle Spectra and LHC Signatures for Large Volume String Compactifications," JHEP 0708 (2007) 061 arXiv:0704.3403 [hep-ph]].

24. S. Iso, N. Okada and Y. Orikasa, "Classically conformal $B^{-}$L extended Standard Model," Phys. Lett. B 676, 81 (2009) arXiv:0902.4050 [hep-ph]], "The minimal B-L model naturally realized at TeV scale," Phys. Rev. D 80, 115007 (2009) arXiv:0909.0128 [hep-ph]].

25. L. A. Anchordoqui, H. Goldberg and T. R. Taylor, "Decay widths of lowest massive Regge excitations of open strings," Phys. Lett. B 668, 373 (2008) arXiv:0806.3420 [hep-ph]].

26. S. Chatrchyan et al. [CMS Collaboration], "Search for narrow resonances using the dijet mass spectrum in pp collisions at sqrt(s) =8 TeV," arXiv:1302.4794 [hep-ex].

27. CMS Report CMS-PAS-B2G-12-014 (2013).

28. B. Hassanain, J. March-Russell and J. G. Rosa, "On the possibility of light string resonances at the LHC and Tevatron from Randall-Sundrum throats," JHEP 0907, 077 (2009) arXiv:0904.4108 [hep-ph]]. 